\title{
Emigrazione economica dei giovani polacchi come fattore significativo di sfide all'interno del matrimonio e della famiglia nella perspettiva canonistica
}

\section{Economic emigration of young Poles as a significant factor of challenges within marriage and family - a canon law perspective}

\begin{abstract}
In Poland, in the last 25 years, theologians, lawyers, sociologist and psychologists have discussed significant changes in the field of marriage and family. The political transformations caused not only economic development, but also cultural transformation and globalization of social phenomena. Among such issues there is the problem of the emigration of young polish citizens. Apart from the positive economic and cultural aspects of working abroad, some negative influence on the strength and unity of the families has been observed. In the article effort was made to examine the impact of labor migration on the reorientation of the general family in the light of matrimonial nullity processes, where the emigrant was involved as the Petitioner or the Respondent party. The author's conclusion is that it is the last chance to create and put into practice, as soon as possible, the modern and interdisciplinary program of pastoral care directed to the families of the migrants, especially the new generation.
\end{abstract}

\section{Keywords}

Labor migration of young Poles, marriage, sociology of family, Roman Catholic faithful, pastoral care for the emigrants, matrimonial nullity processes. 


\section{Introduzione e prezentare il problema}

Il processo matrimoniale è una cartina di tornasole delle disfunzioni e delle patologie della vita coniugale. Alcune analogie sono la testimonianza dellesistenza di certi fatti ed eventi sociali osservabili fin dall'inizio, perché il diritto canonico è una realtà sociale della Chiesa. In questa breve relazione vorrei toccare uno dei problemi legate ai cambiamenti nel matrimonio e nella famiglia che si verificano davanti ai nostri occhi nella Polonia. L' eco di questi cambiamenti si ritrova nei processi dei tribunali ecclesiastici nelle cause di nullità matrimoniale. ${ }^{1}$

Dal giorno dell'adesione della Polonia all'Unione Europea, nei primi 26 mesi più di un milione di polacchi hanno lasciato il paese in cerca di lavoro nei paesi della cosiddetta "vecchia Europa". I sociologi richiamano l'attenzione sull'eterogeneità della società di emigrati, che può essere divisa in tre gruppi: ${ }^{3}$

Il primo gruppo è costituito soprattutto da donne e uomini di mezza età in possesso di basse qualifiche professionali che viaggiano per avere un lavoro stagionale in Germania, Austria, i Paesi Bassi e Spagna. Il lavoro gli occupa 12-18 settimane caratterizzate da uno sforzo intenso al quale fa seguito un ritorno nel proprio paese di 2-6 settimane. Questo tipo di impiego, attualmente conforme alla legge, è nato dall'emigrazione illegale degli anni ' 80 e '9o. Come hanno dichiarato in questionario, i loro guadagni erano destinati soprattutto ad assicurare i bisogni fondamentali della famiglia, tra i quali il finanziamento dell'educazione dei figli. I soldi in eccesso venivano investiti nel settore immobiliare in Polonia (la costruzione di casa, ristrutturamento di appartamento o case) ed erano destinati all'acquisto di beni di consumo (soprattutto l'acquisto di TV

${ }^{1}$ L 'autore lavora come defensore del vincolo presso Tribunale Metropolitano a Cracovia.

${ }^{2}$ Esclusa la Norvegia - [circa 85.000 emigrati polacchi nel anno 2016] e gli operai stagionali che sono persone che lavorano all'estero meno di 12 settimane. I dati statistici della presente relazione provengono dai dati del GUS preparati il 16.10.2017 e pubblicati in internet sul sito ufficiale dell'agenzia www.stat.gov.pl. [ultimo accesso 30 novembre 2017]. Per i dati più specifici vedi l’articolo scritto da Małgorzata Ćwiek e Paweł Ulman, i sociologi dell'Università Economica di Cracovia, Emigracja Polaków po wstąieniu Polski do Unii Europejskiej, à Prawne i ekonomiczne aspekty migracji, ed. P. Kroczek, M. Butrymowicz, Kraków 2016, p. 30-49; DOI: http.//dx.doi.org/10.15633/9788374385473.03.

${ }^{3}$ Cf. M. Okólski, Polityka migracyjna jako instrument promocji zatrudnienia i ograniczania bezrobocia, in: „Biuletyn Migracyjny. Dodatek” (2008) nr. 18, pp. 1-8. Anche: H. Grzymała Mączyńska, Potrzeby nowych kompetencji zawodowych psychologów w związku z polska sytuacja migracyjna, in: Drogi i rozdroża. Migracje Polaków w UE po 1 maja 2004. Analiza psychologiczno-socjologiczna, ed. H. Moszczyńska, A. Kwiatkowska, J. Roszak, Kraków 2010, p. 353. 
e di apparecchi elettronici). Il tratto caratteristico di questa fascia di lavoratori all'estero è la scarsa assimilazione con il paese del lavoro (anche con la sua lingua e la cultura). In molti casi, anche dopo molti anni di lavoro, tali lavoratori non conoscono la lingua del paese di destinazione in modo tale da consentirne un utilizzo quotidiano.

Al secondo gruppo, arrivato come l'ultimo dopo il 2004, appartengono i giovani con una buona istruzione che decidono di emigrare in Gran Bretagna e Irlanda. Sulle isole svolgono lavori al di sotto delle proprie qualifiche professionali, sebbene la conoscenza dell'inglese e la laurea aumentino le opportunità di ottenere una promozione. Sono i maggiori beneficiari dell'apertura dei mercati occidentali alla manodopera polacca. Tuttavia questo gruppo subisce gli effetti del problema principale degli emigrati polacchi nella fascia d'età 2535 anni - età in cui di solito si formano e si sviluppano rapporti interpersonali stabili quali il matrimonio - ossia la scioltezza dei legami familiari con quelli rimasti in Polonia.

Il terzo gruppo include gli studenti che decidono di restare per un certo periodo nel paese di studio nellambito di programmi universitari di scambio. Il gruppo di cui sopra presenta dei tratti sociali tipici della sua età: è disposto a fare conoscenze in un continente sempre più multiculturale ad ovest e decide di avere relazioni intime di carattere emotivo sia con studenti polacchi che con altri cittadini dell'UE di diversa origine etnica.

Il quarto gruppo di emigrati è rappresentato invece da coloro che decidono di partire per gli Stati Uniti. In Polonia esiste una lunga tradizione migratoria verso l'America, con una forte componente di emigrati in partenza dal sud-est della Polonia. Ė un gruppo di lavoratori forte e unito in cui spesso i membri della famiglia si alternano nei loro viaggi. Negli anni ' 80 del XX secolo l’emigrazione oltreoceano implicava una lunga separazione, per diversi anni, dal proprio paese e dalla famiglia. È proprio tra la popolazione di emigrazione americana che si registra il maggior numero di casi di separazione forzata, un fenomeno che può durare anche più di un decennio dando in alcuni casi vita a relazioni di tipo bigamo. Gli emigrati negli USA sono molto attivi nei tribunali vescovili attraverso richieste di nullità dei matrimoni contratti in Polonia. Tuttavia l'esperienza giudiziaria ${ }^{4}$ dimostra che nella maggior parte dei casi i motivi di separazione non rientrano nella casistica dei fattori oggettivi elencati nel codice

${ }^{4}$ Ricerche condotte dall'autore del presente saggio sulle cause indirizzate al Tribunale Metropolitano a Cracovia di I e II istanza. 
di diritto canonico (gli ostacoli matrimoniali, i difetti del consenso matrimoniale, la mancanza di forma canonica) all'origine dell'annullamento del matrimonio, ma i problemi matrimoniali preesistenti all'emigrazione; l'emigrazione ha avuto l'effetto di accelerare il processo di risoluzione del rapporto.

\section{Gli effetti dell'emigrazione per i matrimoni e le famiglie}

Alla luce dello sviluppo economico del paese gli analisti del governo hanno riscontrato degli aspetti positivi legati allemigrazione. Secondo gli esperti del NBP (Banca Centrale Polacca) nel 2007 il trasferimento di fondi verso Polonia ha raggiunto grazie all'emigrazione la cifra di 5 milioni di euro, il doppio rispetto all'anno 2004. Tali entrate erano possibili anche grazie al fatto che gli emigrati erano in gran parte lavoratori stagionali che hanno investito i soldi guadagnati all'estero nel paese di origine.

Professoressa Zofia Kawczyńska-Butryn dall'Università di Maria Skłodowska - Curie a Lublin, specialista in sicenze sociali, ha condotto delle ricerche approfondite sull'atteggiamento dei genitori nell'ambito dei fenomeni di emigrazione dalle regioni più povere della Polonia, che detengono il primato delle partenze all'estero. I risultati di questa ricerca hanno dimostrato che sono soprattutto le donne (a differenza degli uomini più attive, meno depressive e affette da dipendenze) a correre il rischio di andare all'estero per motivi di lavoro, lasciando spesso i figli in Polonia. ${ }^{5}$

Si tratta di un vero e proprio "segno del tempo", una testimonianza dei cambiamenti contemporanei e della ridefinizione del ruolo della donna nel matrimonio, specialmente nelle zone rurali della Polonia centrale e settentrionale, tradizionalmente dominata da una struttura patriarcale. ${ }^{6}$ Purtroppo tale fenomeno di emigrazione femminile, descritto dalla prof. Kawczyńska-Butryn è all'origine di un fenomeno sociale definito "maternità transfrontaliera,", probabilmente l'effetto collaterale più drammatico legato all'emigrazione. I media, gli ambienti legati agli studi sulla famiglia, nonché coloro che lavorano nei servizi

${ }^{5}$ Si stima che negli anni 2004-2006 circa il 28-33\% delle emigrate abbia lasciato a casa i figli con meno di 7 anni.

${ }^{6}$ Cf. Z. Kawczyńska-Butrym, Migracje, wybrane zagadnienia, Lublin 2009, pp. 38-49.

7 Quest' espressione proviene dallo studio di S. Urbańska: Transnarodowość jako perspektywa ujęcia macierzyństwa w warunkach migracji, in: Migracje kobiet. Perspektywa wielowymiarowa. ed. K. Slany, Kraków 2008, p. 78. 
sociali hanno registrato delle storie di bambini lasciati in Polonia al genitore meno competente ed incapace di assicurare un sostegno sociale ed emotivo alla prole. ${ }^{8}$

Il sacerdozio degli emigrati ha cominciato ad agire in modo tempestivo ed efficace nelle città caratterizzate dalla presenza di una forte minoranza polacca. Tuttavia se si guarda all'emigrazione considerando il numero di processi in corso nei tribunali vescovili polacchi sembra che le attività formative di preparazione al matrimonio siano state messe da parte. Mi permetto di sostenere che il problema dell'emigrazione sia stato sottovalutato, mentre le persone che partivano dalla Polonia avevano di solito meno di 35 anni. Alla luce dei dati statistici rilevati in precedenza relativi all'aumento dell'età dei nubendi è logico trarre la conclusione che una parte dei partecipanti dei corsi prematrimoniali decida oppure abbia già deciso di emigrare in concomitanza del matrimonio. Tale evento risulta spesso troppo difficile, per la continuazione del matrimonio.

Molte persone che hanno deciso di emigrare non si rendono conto dei costi emotivi e sociali di tale viaggio. Ciò vale ancora di più per coloro i quali hanno lasciato nel paese di partenza il proprio coniuge e/o i figli. Alcuni ritengono (una parte nè ancora convinta) di poter preservare il ruolo genitoriale mantenendo allo stesso tempo un contatto quotidiano con il coniuge attraverso videochiamate via internet, contatti telefonici, corrispondenza via e-mail. Tuttavia la vita sospesa tra due mondi, la mancanza di appartenenza alla società locale influisce in modo negativo sull'emigrato e gli fa sentire di più il senso di separazione. Una ricerca indipendente condotta dalla giovane psicologa, dotoressa Marta Głowacka, tra gli emigrati londinesi ha dimostrato che anche in presenza di un buon livello di intimità nei momenti di rari incontri tra i coniugi un impegno quotidiano troppo debole nel rapporto ha causato una graduale distruzione dei legami interpersonali dei coniugi divisi. ${ }^{9}$ Lautrice ne ha analizzato gli effetti prendendo in considerazione un intervallo di separazione di circa 3-4 anni. ${ }^{10}$

${ }^{8}$ Le indagini rivelano che il genitore che trova lavoro da emigrato sia dotato di più capacità sociali mentre quello meno operativo rimane a casa con i bambini. Cf. E. Winnicka, Eurosieroty „Polityka” 46 (2629) del 17 novembre (2007), p. 34.

9 Cf. M. Głowacka, Dynamika kontaktów seksualnych i ich postrzegany wpływ na jakość związku w sytuacji długofalowej rozłąki - studium przypadku, Poznań 2007, una parte dello studio è disponibile on-line sul sito della Filiale londinese dell' Associazione Polacca di Psicologia (www.polishpsychologistsclub.org.) (30.11.2017).

${ }^{10}$ Cf. A. Jucewicz SVD, Skutki moralne współczesnej migracji zarobkowej, in: Ludzie $w$ drodze, ed. D. Cichy SVD, Warszawa 2012, pp. 97-99. 
Le cause di nullità del matrimonio tra i lavoratori emigrati costituiscono una prova delle lacune legate allesperienza del fidanzamento. Analizzando le cause dei matrimoni che sono finiti, si può riscontrare la bassa qualità della formazione al matrimonio, unattività spesso sottovalutata non solo dai fidanzati ma anche dai sacerdoti. Una mancanza ancora più grave nel caso in cui uno dei fidanzati sia già un emigrato oppure abbia in cantiere il progetto di andare allestero dopo il matrimonio. Nei casi in cui si richiede una partecipazione al corso in anticipo, spesso è un'altra persona (fratello/sorella oppure un amico/un'amica) che sostituisce il fidanzato/la fidanzata assente nelle attività di preparazione al matrimonio. I giovani adulti invece di considerare il fidanzamento unoccasione per conoscere meglio il carattere, le caratteristiche comportamentali dell'altro nonché uno strumento utile a fare piani e a prendere le prime decisioni sulle nozze sono portati a sottovalutarlo non senza una certa ingenuità. Essi sono convinti che il loro rapporto sia abbastanza forte da sopravvivere a distanza, basandosi sulle soddisfazioni materiali legate alla sfera sessuale e al pensiero dei soldi guadagnati.

La realtà matrimoniale che richiede la realizzazione degli impegni legati al nuovo ruolo sociale (ad es. il trasloco a casa dei genitori di uno degli sposi e la necessità di vivere con i suoceri), la mancanza di un sostegno - della presenza fisica del marito/della moglie; il senso di solitudine e di dipendenza economica dal coniuge che lavora allestero, ma anche la stanchezza e la gelosia; tutto questo causa insoddisfazione e genera frustrazione e scoraggiamento nel coniuge. A ciò si aggiungono problemi di natura intima, l'irregolarità di contatti sessuali (un elemento importante dellamore coniugale), le aspettative gonfiate e non realizzate nei confronti dell'altro e una sfiducia progressiva. Durante i momenti fugaci della vita di coppia nel paese di origine gli sposi consacrano a se stessi e al loro rapporto meno tempo di quello che vorrebbero e dovrebbero dedicare.

\section{Qualche questioni dall’ esperienza dell'autore}

Nel tribunale Metropolitano di Cracovia di I e II istanza sono due le tipologie di cause dominanti tra gli emigrati: la prima è rappresentata dalle vittime ovvero i giovani adulti emigrati nei paesi dell'Europa Occidentale e negli USA. La seconda può essere considerata un' "effetto boomerang" della prima, è rappresentata dalle conseguenze di una lunga separazione dei coniugi e della mancata realizzazione degli impegni genitoriali da parte del padre o della madre. 
L' emigrazione di uno dei genitori (o addirittura di entrambi) nel contesto del funzionamento sociologico e psicologico della famiglia, indipendentemente dai benefici di natura economica, lascia il bambino in una situazione emotivamente instabile rispetto ai suoi coetanei che hanno una famiglia unita. Tali condizioni influenzano in modo negativo lo sviluppo e la formazione del bambino, esponendolo al rischio di sviluppare una personalità disfunzionale in età adulta, incapace di costruire un rapporto duraturo con una altra persona.

Anche se dal 2008 il numero degli emigrati rientrati in Polonia è in aumento, ${ }^{11}$ tuttavia le conseguenze del rientro sono destinate ad influenzare la vita familiare dei coniugi anche dopo il rientro. Per questo ritengo essenziale l'elaborazione di nuovi programmi per i corsi per i fidanzati che tengano conto delle problematiche specifiche legate al lavoro all'estero. Specialmente nelle diocesi più esposte a fenomeni di emigrazione bisognerà poi mettere in preventivo anche le difficoltà riscontrate nel processo di costruzione di una famiglia per le vittime dei cambiamenti economici nella regione. Secondo il principio di cooperazione della fede e della ragione suggerirei di utilizzare nel modo più ampio possibile i risultati delle ricerche psicologiche e sociologiche sugli effetti dei fenomeni di emigrazione (oppure di una lunga separazione in generale) tra i neo sposi. Bisognerà poi incoraggiare i partecipanti a cercare soluzioni alternative con un impatto emotivo più contenuto sulla vita di coppia come la partenza di entrambi gli sposi o dei genitori con i figli. Si è visto come nei casi di un soggiorno più lungo 4nei paesi di destinazione (per es. la Gran Bretagna e i cittadini delle sue ex-colonie), nonostante i vantaggi economici siano più bassi rispetto ai "single" in emigrazione, le famiglie emigrate al completo hanno affrontato meglio la crisi di identità della relazione. ${ }^{12}$

${ }^{11}$ Nel 2008 il numero dei polacchi che hanno scelto di emigrare nei paesi della cosiddetta "vecchia Europa" ammontava a 1,820,000 individui. Nel 2009, per la prima volta dopo 5 anni questo numero è calato a 1,570,000, (la fonte: GUS). Migracje powrotne Polaków. Powroty sukcesu czy rozczarowania?, K. Iglicka (ed.), Warszawa 2002.

12 Nel 2009 il 20\% degli emigrati era costituito da famiglie. Ovviamente l'immersione nel nuovo ambiente dei bambini e il lavoro dei genitori spesso fino a tardi esponevano i figli a problemi di ambientamento, esclusione sociale, nostalgia del paese e della famiglia del paese di partenza (soprattutto i nonni). Tuttavia l'emigrazione di intere famiglie si è dimostrata la scelta vincente per garantire una relazione coniugale stabile e per instaurare un rapporto solido tra genitori e figli. Nel 2009 dott. Katarzyna Czepiel ha fatto le sue recherche sulle famiglie polacche in Irlanda: Rola rodziny w kształtowaniu tożsamosci dziecka na emigracji, in: Rodzina Polska na emigracji $w$ duszpastersko-społecznej refleksji, I. Celary, G. Polok (eds.), Katowice 2011, pp. 140-145. 
Ritengo che sia altrettanto importante di intensificare i momenti di dialogo tra gli aspiranti sposi e il sacerdote chiamato a registrare il protocollo canonico nella situazione in cui uno dei due fidanzati indichi come luogo di residenza un paese straniero. Bisogna sempre capire se una lunga separazione dopo il matrimonio (rischiosa ai fini del consolidamento della relazione) sia oggettivamente necessaria - per es. causata dalla mancanza di lavoro e di prospettive professionali - oppure se si tratta soltanto di un elemento di avidità e di sovrapposzione dell'avere (ad es. un recinto alla moda, un cortile di granito) all'essere. L'obbligo del sacerdote non è solo quello di ricordare al futuro emigrato i suoi doveri religiosi nel paese di emigrazione, ma anche di metterlo in condizione di compiere le pratiche religiose e di mantenere un legame con la comunità della Chiesa cattolica. Tale obiettivo può essere raggiunto mettendo a disposizione i recapiti delle istituzioni ecclesiastiche della diaspora polacca, delle filiali della Missione Cattolica Polacca ${ }^{13}$, delle associazioni attive presso le diocesi locali, delle organizzazioni di beneficenza ${ }^{14}$ ecc. Sebbene queste informazioni siano disponibili su internet - l'esperienza dei sacerdoti suggerisce che i tentativi spontanei di cercare Dio nel bel mezzo di un'attività lavorativa e di nuove condizioni del paese culturalmente estraneo emergano solamente nella situazione di crisi, di trauma o che siano comunque dettate dalla necessità di espletare una funzione (ad es. quella di padrino o madrina). ${ }^{15}$

Finalmente dal punto di vista di una persona che lavora in tribunale, mi sembra che sarebbe una buona prassi giudiziaria in stabilire una cooperazione continua (attraverso diversi eventi publici, bilaterali e anche privati) con i tribunali delle diocesi in cui si trova il maggior numero degli immigrati polacchi. Questa idea sarebbe permettere una migliore conoscenza dei vari tribunali e la condivisione delle esperienze.

${ }^{13}$ Cf. I. Celary, G. Potok, Rola kapłana jako duszpasterza rodzin polskich na emigracji, in: Rodzina polska na emigracji w duszpastersko-społecznej refleksji, (eds.) I. Celary, G. Potok, Katowice 2011, pp. 86-89.

${ }^{14}$ Cf. L'istituzione che vale la pena di annotare è Polish Psychologists Club con sede a Londra (http://www.polishpsychologistsclub.org/ (30.11.2017)). Si tratta di un'associazione di specialisti polacchi attivi nei settori di psicologia, pedagogia e di sociologia utile ai propri connazionali attraverso un sostegno psicologico e pedagogico gratuito. Tale organizzazione attiva in Gran Bretagna tramite le sue filiali ha condotto numerosi studi sui fenomeni di emigrazione.

15 Cf. J. Poznański, Małżeństwo i rodzina w czasach migracji, "Posłaniec" 9 (2008) articolo disponibile anche on-line: www.opoka/64.233.183.104/search?q=cache:SFtcfxJW (30.11.2017). Le brave richerche sociologiche si trovano in libro di J. Mariański: Wiara i wierzenia Polaków w niestabilnej nowoczesności. Analiza socjologiczna, Lublin 2014, p. 39-51. 


\section{Invece di conslusione: la spiegazione metodologica}

Il diritto canonico al contrario delle discipline scientifiche, è aperto alle esperienze delle altre scienze, particolare scienze mediche, psichologice e delle scienze sociali. Il professore Sobanski, in suo libero Metodologia del diritto canonico ha scritto: "Sulla selezione dei metodi non decide qualche principio astratto, ma il tentativo di trovare delle soluzioni concrete a dei problemi reali la cui trattazione rientra nel diritto ecclesiastico. Le istituzioni e i regolamenti giuridici sono sempre un frammento di una realtà sociale che resta decisamente complessa. Il canonista dovrà interpretarla in una misura necessaria alla risoluzione di una problematica canonica completa". ${ }^{16} \mathrm{Al}$ fine di portare a compimento quel fine fondamentale e supremo conosciuto come bene degli animi il diritto canonico va esaminato in diversi aspetti. Seguendo tale approccio ho scelto di rinunciare ad un metodo canonico "puro" con laugurio che in questo motivo il problema sarà mostrato da una prospettiva più ampia.

\section{Bibliography}

Central Statistc Office of Poland: http://stat.gov.pl/files/gfx/portalinformacyjny/pl/defaultaktualnosci/5471/2/10/1/informacja_o_rozmiarach_i_kierunkach_emigracji_z_ polski_w_latach_20042016.pdf (30.11.2017).

Czepiel K., Rola rodziny $w$ kształtowaniu tożsamosci dziecka na emigracji, pp. 140-145, in: Rodzina Polska na emigracji w duszpastersko-społecznej refleksji, (ed.) I. Celary, G. Polok, Katowice 2011.

Ćwiek M., Paweł Ulman, Emigracja Polaków po wstąpieniu Polski do Unii Europejskiej, p. 30-49; DOI: http.//dx.doi.org/10.15633/9788374385473.03, in: Prawne i ekonomiczne aspekty migracji, (eds.) P. Kroczek, M. Butrymowicz, Kraków 2016.

Głowacka M., Dynamika kontaktów seksualnych i ich postrzegany wpływ na jakość związku w sytuacji długofalowej rozłąki - studium przypadku, Poznań 2007, http:// www.polishpsychologistsclub.org (30.11.2017).

Iglicka K. (ed.) Migracje powrotne Polaków. Powroty sukcesu czy rozczarowania?, Warszawa 2002.

Jucewicz A., SVD, Skutki moralne współczesnej migracji zarobkowej, in: Ludzie w drodze, (ed.) D. Cichy SVD, Warszawa 2012, pp. 95-106.

Kawczyńska-Butrym Z., Migracje, wybrane zagadnienia, Lublin 2009.

16 R. Sobański, Metodologia prawa kanonicznego, Katowice 2004, p. 25. 
Mariański J., Wiara i wierzenia Polaków w niestabilnej nowoczesności. Analiza socjologiczna, Lublin 2014.

Sokólski M., Polityka migracyjna jako instrument promocji zatrudnienia i ograniczania bezrobocia, „Biuletyn Migracyjny. Dodatek” (2008) 18, pp. 1-8.

Grzymała - Mączyńska H., Potrzeby nowych kompetencji zawodowych psychologów $w$ związku z polska sytuacja migracyjna, in: Drogi i rozdroża. Migracje Polaków w UE po 1 maja 2004. Analiza psychologiczno-socjologiczna, (eds.) H. Moszczyńska, A. Kwiatkowska, J. Roszak, Kraków 2010, pp. 353-368.

Poznański J., Małżeństwo i rodzina w czasach migracji, "Posłaniec" 9 (2008), www. opoka/64.233.183.104/search?q=cache:SFtcfxJW (30.11.2017).

Sobański R., Metodologia prawa kanonicznego, Katowice 2004.

Celary I., Potok G., Rola kapłana jako duszpasterza rodzin polskich na emigracji, in: Rodzina polska na emigracji $w$ duszpastersko-społecznej refleksji, (eds.) I. Celary, G. Potok, Katowice 2011, pp. 80-90.

Urbańska S., Transnarodowość jako perspektywa ujęcia macierzyństwa $w$ warunkach migracji, in: Migracje kobiet. Perspektywa wielowymiarowa, (ed). K. Slany, Kraków 2008, pp. 75-88.

Winnicka E., Eurosieroty, „Polityka” 46 (2629) 17.11 2007, p. 34. 